\title{
Pandemia por COVID y el síndrome de duelo: ¿un enemigo reemergente en la salud mental?
}

\author{
COVID pandemic and grief disorder: A reemerging enemy in mental health? \\ C. Cecilia Flores-Ruiz ${ }^{1, a}$, Tatiana Leonela Cuba-Llanos ${ }^{1, a}$, W. Samir Cubas ${ }^{2, b}$
}

\section{Señores Editores:}

La pandemia por COVID-19 viene generando una crisis en la salud pública de nuestro país, causando una mortalidad del 9,42\%, siendo Lima Metropolitana, la primera región que presenta una alarmante cifra de 78 564 fallecidos hasta la fecha (1). Las cifras reflejan un incremento del número de fallecidos en las regiones de la costa como Lima, Callao, Arequipa y La Libertad en relación al resto de regiones del Perú, esto debido a que se encuentra una mayor concentración de la población en estos departamentos. A más de un año de haber confirmado el primer caso de coronavirus en nuestro país, del inicio del estado emergencia y de la implementación de las nuevas medidas de higiene, las cifras de mortalidad ascienden a 188708 fallecidos y el número de defunciones se ha triplicado en tan solo 8 meses $(1,2)$.

Estas cifras asociadas a la morbimortalidad del COVID-19, han generado un reemergente síndrome de duelo a nivel mundial. Este se define como el proceso de adaptación emocional que atraviesa una persona ante una pérdida de un empleo, vivienda y/o ruptura de una relación, según el Ministerio de Salud del Perú a través de su plan de salud mental, 2020 - 2021 (3). Por otro lado, según el DSM-5, lo define únicamente como el proceso ante la pérdida de un ser querido (4). Se ha reportado cifras de síndrome de duelo en el contexto de la pandemia por COVID-19 que oscilan el 9,8\% en países como Estados Unidos de América
(5). Recientes estudios han descrito que el desarrollo de este síndrome de duelo durante la pandemia por COVID-19, se encuentra asociado principalmente a la exposición de las personas a constantes pérdidas humanas, obteniendo una elevada tasa de casos de este síndrome (60\%). Asimismo, la presencia de condiciones mentales previas está ligado al progreso del duelo normal a patológico (40\%) (6).

Esta no es la única condición mental que se asocia a la pandemia por COVID-19, también se han reportado tasas elevadas de ansiedad $(28,8 \%)$, estrés $(8,1 \%)$, depresión mayor (16,5 - 29.9\%), etc.; siendo factores de riesgo para el desarrollo del síndrome de duelo (3). Por otro lado, la resolución del duelo a través de la realización de intervenciones oportunas y acompañamiento psicoterapéutico de profesionales de salud es limitada debido a la restricción de la comunicación interpersonal y la adaptación de cambios en la rutina diaria de las personas producto del confinamiento y el distanciamiento social producido por la pandemia del COVID $19(7,8)$.

Actualmente, las estrategias psicoterapéuticas para el manejo del duelo son: la Terapia cognitivoconductual, Terapia del duelo complicado (TDC) y Terapia interpersonal, estas se enfocan en los procesos relacionados con la pérdida y se centran en los síntomas de los recuerdos intrusivos dolorosos, facilitando la recuperación, preservando la calidad, deteniendo el deterioro funcional y reduciendo los

Facultad de Medicina Humana, Universidad Nacional de San Martín. Tarapoto, Perú.

2 Departamento de Cirugía Torácica y Cardiovascular, Hospital Nacional Edgardo Rebagliati Martins.

Lima, Perú. 
comportamientos de alto riesgo que afectan la calidad de sueño y la integridad mental del individuo (9).

En la mayoría de los casos, la intervención de psicofármacos como una medida terapéutica no es necesaria, a menos que se presenten trastornos psiquiátricos de fondo (depresión, demencia, etc.), donde su empleo se vuelve imperativo. En un reciente estudio triple ciego, aleatorio y controlado sobre el tratamiento farmacológico del duelo prolongado realizado en la ciudad de Nueva York en los Estados Unidos de América se ha reportado el uso de fármacos como antidepresivos tricíclicos y naltrexona, en pacientes que ameritaba su uso, además se hizo hincapié en evitar la interacción total de estos medicamentos con las benzodiacepinas (9).

Este reemergente síndrome está considerado en los programas de seguimiento familiar con pérdidas mortales por el COVID-19. Además de evaluar a profundidad la problemática, debemos de tratar de resolver los procesos de pérdida a través de la psicoeducación, discusión de recuerdos positivos y negativos del difunto, reestructuración cognitiva de los pensamientos erróneos, empleando la terapia narrativa (10).

La terapia narrativa permite abordar a tiempo comportamientos evitativos y participaciones inadecuadas en actividades cotidianas que antes eran agradables para la persona, a través de herramientas como el discurso del duelo y la deconstrucción, lo que permite a la persona explorar y ampliar sus puntos de vista sobre la situación de pérdida que ha sufrido, aliviando episodios de empeoramiento agudo del dolor, y en paralelo, potencia y trabaja la aceptación de la pérdida, a través de la construcción de nuevos vínculos con lo perdido (10).

\section{Correspondencia:}

Carmen Cecilia Flores Ruiz

Facultad de Medicina Humana, Universidad Nacional de San Martín, Tarapoto, Perú. Jr. Amorarca 315, Tarapoto, Perú.

Teléfono: 51969404825.

Correo electrónico: ceciliaafr10@gmail.com

Fuente de financiamiento: Autofinanciado

Declaración de conflicto de intereses: Los autores declaran no tener conflicto de intereses.

\section{REFERENCIAS BIBLIOGRÁFICAS}

1. Ministerio de Salud. Sala Situacional COVID-19 Perú. Lima: Ministerio de Salud; 2020. (Citado el 15 junio del 2021). Disponible en: https://covid19. minsa.gob.pe/sala_situacional.asp

2. Flores MG, Soto A, De La Cruz-Vargas JA. Regional distribution of COVID-19 mortality in Peru. RFMH URP. 2020; 24; 21(2), 326-34. DOI: 10.25176/ RFMH.v21i2.3721

3. Cutipe Y, Mendoza M, Uribe R, et al. Plan de salud mental, 2020 - 2021 (en el contexto COVID-19). Lima: Ministerio de Salud; 2020. (Citado el 15 junio del 2021). Disponible en: http://bvs.minsa.gob.pe/ local/MINSA/5092.pdf

4. Asociación Americana de Psiquiatría. Manual diagnóstico y estadístico de los trastornos mentales (DSM-5®). 5a Ed. Arlington, VA: Asociación Americana de Psiquiatría; 2018.

5. Tang, S, Xiang, Z. Who suffered most after deaths due to COVID-19? Prevalence and correlates of prolonged grief disorder in COVID-19 related bereaved adults. GH. 2021; 17(1):1-9. DOI: 10.1186/s12992-02100669-5

6. Killikelly C, Smid GE, Wagner B, Boelen PA. Responding to the new International Classification of Diseases-11 prolonged grief disorder during the COVID-19 pandemic: a new bereavement network and three-tiered model of care. Public Health. 2021;191:85-90. DOI: 10.1016/j.puhe.2020.10.034

7. Higgins MO, García O, Torales J. Efecto de la cuarentena por la pandemia de COVID-19 en la salud mental. Academic Disclosure. 2021; 1(2): 1521. (Citado el 15 junio del 2021). Disponible en: https://revistascientificas.una.py/index.php/rfenob/ article/view/122/122

8. Huarcaya-Victoria, J. Consideraciones sobre la salud mental en la pandemia de COVID-19. Rev Peru Med Exp Salud Publica. 2020; 37(2): 327-34. DOI: 10.17843/rpmesp.2020.372.5419

9. Gang J, Kocsis J, Avery J, et al. Naltrexone treatment for prolonged grief disorder: study protocol for a randomized, triple-blinded, placebo-controlled trial. Trials. 2021; 22(110): 15. DOI: 10.1186/s13063-02105044-8

10. Massa E. Duelo patológico, tratamiento con psicoterapia narrativa. REPI. 2017; 20(1): 321 - 37. (Citado el 16 agosto del 2021). Disponible en:https:// www.medigraphic.com/pdfs/epsicologia/epi-2017/ epi171q.pdf

Recibido: 01/07/2021

Aceptado: 16/09/2021 